Published in Colloids and Surfaces B: Biointerfaces (2004) 33(3-4):251-258

\title{
Water Vapor Sorption by the Pedal Mucus Trail of a Land Snail
}

\author{
B.J. Lincoln, T.R.E. Simpson, and J.L. Keddie* \\ Department of Physics, University of Surrey, Guildford, Surrey GU2 7XH, UK
}

\begin{abstract}
The hydrophilicity of pedal mucus trails deposited by marine snails influences the settlement of other organisms and can potentially influence the trailing and homing mechanisms of terrestrial snails. The composition of pedal mucus deposited as a trail on a solid substrate by the giant African land snail (Achatina marginata) has been probed non-invasively using infrared ellipsometry. The primary chemical groups in the mucus (in its native state) have been identified through their characteristic infrared absorption frequencies. Water vapor sorption in the mucus trails in equilibrium with the atmosphere was measured as a function of the relative humidity $(\mathrm{RH})$. When $\mathrm{RH}=84 \%$, the mucus contains 53 volume percent water. The water sorption isotherm of the mucus trail can be described through a Flory-Huggins polymer/solvent interaction parameter of $\chi=0.54$ \pm 0.1 , which is comparable to the value for some synthetic hydrophilic polymers, such as poly(vinyl pyrrolidone).
\end{abstract}

Key words: gastropod; hydrophilicity; mucus; infrared ellipsometry; water sorption

\footnotetext{
* Corresponding author. Telephone: +44-1483-686803; Facsimile: +44-1483-686781; e-mail: j.keddie@surrey.ac.uk
} 
Published in Colloids and Surfaces B: Biointerfaces (2004) 33(3-4):251-258

\section{Introduction}

Gastropods, such as slugs and snails, secrete a trail of mucus (i.e. a "snail trail") from their pedal gland while travelling across a surface [1]. The unique mechanical properties of snail pedal mucus enable the animal's locomotion while also causing the mucus to function as an adhesive to the substrate [2]. The mucus trail performs a number of other functions [1], including the provision of mechanisms for re-tracing a path (i.e. "homing") and for finding a mate of the same species by following a trail [3]. An understanding of the functionality of trail mucus, including its interactions with water vapor, can therefore lead to a means of controlling the reproduction of snails and thereby limiting their impact on the environment, especially vegetable crops [4]. It has been reported [5] that the presence of a marine snail's pedal mucus on a surface influences the settlement of other adhering marine organisms (such as barnacles) to that surface. The hydrophilicity or hydrophobicity of a substrate, in particular, has been found to influence the settling of other organisms [5-7], which suggests a reason why a mucus trail will have an impact.

When freshly deposited by terrestrial snails, trails of pedal mucus are reported to be in the range of 10 to $20 \mu \mathrm{m}$ thick [8]. But since the mucus typically consists of between 90 and 99.7\% water by weight [1], the trails dry to leave a much thinner solid film. The main constituent of gastropod mucus is a complex of proteins and polysaccharides. This complex is usually classified into the broad categories of mucopolysaccharides and glycoproteins. The chemical compositions of the mucus of several species of molluscs have been determined [1]. 
The technique of SDS-PAGE electrophoresis has been applied successfully to the trail mucus of seven species of land mollusc for the identification of the chemistry of the constituent polysaccharides and proteins [4]. Two major drawbacks of SDS-PAGE are that it is time-consuming and it can potentially introduce artifacts through extraction and derivation procedures. Compared to SDS-PAGE and similar techniques, IR spectroscopy is attractive for several reasons [9]. It is fast and does not require any sample extraction. Moreover, it avoids the threat of artifacts by allowing analysis of a surface or film in its unaltered, natural state.

Quite recently, the first clear infrared (IR) spectra were reported for the mucus produced by nine different species of gastropods [9]. Transmission IR spectroscopy was employed to detect the characteristic IR absorption bands of the mucopolysaccharides as well as the glycoproteins. Small differences were reported between species, meaning that IR spectroscopy can potentially be used for species identification. A disadvantage of transmission IR spectroscopy is that substrates are limited to materials that are transparent to IR radiation, which excludes many polymer substrates. Previously, an IR spectrum has been obtained from the mucus of Achatina fulica [10], as well as three other species, but the spectra were not analysed in depth.

Infrared spectroscopic ellipsometry (IRSE) is a non-invasive technique that can determine the structure and optical constants of thin films and bulk materials. Most work to-date has been applied to semiconductors. The technique has, however, been shown to 
Published in Colloids and Surfaces B: Biointerfaces (2004) 33(3-4):251-258

be a quantitative tool for the measurement of the concentration of biological molecules in a thin layer [11] and for the determination of the structure of organic films [12]. Unlike transmission methods, IRSE relies on reflection and therefore does not require transparent substrates but can instead be applied to nearly any planar interface. As the technique require neither a baseline correction nor the suppression of absorption bands due to atmospheric $\mathrm{CO}_{2}$ or water [11], IRSE offers a distinct advantage over conventional IR spectroscopy.

This work has two key objectives. One objective is to develop IRSE as a technique to detect terrestrial snail mucus trails in their native state on a solid substrate. This objective is motivated by the need for techniques that are faster than SDS-PAGE electrophoresis and that are not prone to artifacts. A second objective is to characterize the hydrophilicity of the pedal mucus by determining the equilibrium water vapor sorption at constant temperature as a function of the relative humidity. Although the constituent molecules of gastropod mucus are water-soluble when first deposited, the hydrophilicity of mucus in pedal trails has not been characterized.

Previous research characterizing the "wettability" of mucus has only been semiquantitative. Specifically, relative comparisons of the hydrophobicity of pedal mucus trails from marine snails were made via contact-angle measurements [5]. The sorption isotherms presented here enable the prediction of the state of hydration in mucus trails for a given ambient humidity. This quantitative measurement of the hydrophilicity of mucus trails in their as-deposited state has possible relevance to species-specific trailing 
Published in Colloids and Surfaces B: Biointerfaces (2004) 33(3-4):251-258

mechanisms and to the attachment of other organisms to the trails. Although the present work concerns terrestrial snails, the same approach could be applied to marine snails in future work.

\section{Materials and Procedure}

Mucus trails deposited by the giant African land snail (Achatina marginata) were studied. Prior to the experiments, the snails were handled in the laboratory to habituate them with the environment and to minimize their stress. Before the deposition of mucus trails for an experiment, the snail was first allowed to acclimate to the laboratory environment for a few minutes. It was next left to crawl on a smooth silicone surface so that mulch and other debris on its foot were removed. Glass microscope slides coated with a $200 \mathrm{~nm}$ layer of gold were used as substrates for IR ellipsometry. Mucus trails were deposited onto the gold surface by placing the snail at one end and coaxing it to travel across the surface by positioning food at the opposite end. The trail films were then studied without any perturbation or disruption.

The mucus films were analyzed using an FTIR spectroscopic ellipsometer (Model GESP5-FTIR, SOPRA Sa., Bois-Colombes, France). A single IR ellipsometry measurement was acquired within about two minutes, but in order to obtain smoother spectra, an average of 64 cycles was obtained in each measurement, taking approximately two hours in total for the data acquisition. The spectral resolution of the measurements was set to $16 \mathrm{~cm}^{-1}$. 
In measurements of hydrophilicity, IRSE was performed on substrates in an environmental chamber. IR radiation entered and exited the chamber through windows made from $20 \mu \mathrm{m}$ undoped silicon wafers that had been polished on both sides. As silicon is transparent throughout most of the IR range, it is an effective material for windows and is also resistant to moisture, unlike IR-transparent salts such as $\mathrm{KBr}$. The windows were fixed at an angle of $65^{\circ}$ with respect to the normal to the substrate. In visible ellipsometry experiments, similar cells having thin glass windows have been employed [13]. The temperature of the cell was ca. $25^{\circ} \mathrm{C}$.

The humidity was regulated by circulating air with a fixed RH through the chamber using a pump. The air flowed across the surface of supersaturated salt solutions prior to entering the chamber in order to adjust the RH. Aqueous solutions (i.e. slushes) of $\mathrm{LiCl}$, $\mathrm{MgCl}_{2}, \mathrm{NaBr}, \mathrm{NaCl}, \mathrm{KBr}$ and $\mathrm{KCl}$ were used to set the $\mathrm{RH}$ to nominal values of 11.2, 32.8, 57.6, 75.3, 80.9, and 84.3\%, respectively [14]. Care was taken to ensure that crystals did not emerge from the solution and that there were no salt residues on the surfaces of the glassware. An RH of $0.1 \%$ was obtained by passing the air over a silica gel desiccant. A digital hygrometer (Hanna Instruments) showed that the RH was stable to within $0.1 \%$ during a measurement. After the salt solutions were changed, it took approximately two minutes before the humidity stabilized within the sample chamber. Preliminary measurements revealed that when the RH was lowered in the cell, a mucus film dehydrated within minutes. Thereafter, the spectra did not change over time, meaning that the film thickness and composition had stabilized. It was observed that 
when the humidity was increased abruptly, the time to equilibration was longer than when the humidity was decreased.

Simulations of the data were produced with an effective medium approximation (EMA) [15] relying on the optical constants of water in the literature [16] and measured constants for dry mucus. The EMA model predicts the optical constants of a composite material through consideration of the optical constants of each constituent. The optical constants for the dry mucus (i.e. equilibrated in the chamber at $\mathrm{RH}=0 \%$ ) were obtained experimentally. The ellipsometry spectra for a mucus layer of known thickness were fitted using the real and imaginary components of the refractive index at each wavelength as a variable. The error between experimental IRSE data and simulations was minimized using a Levenberg-Marquardt algorithm [17] while varying both the film thickness and the volume fraction of water in the EMA model.

\section{Results and Discussion}

Identification of Chemical Groups in Trail Mucus

Ellipsometry measures the ellipticity, $\rho$, which is defined in terms of the ratio of the Fresnel reflection coefficient in the plane to the coefficient normal to the plane. The greatest sensitivity to a surface layer can often be obtained with ellipsometry through a calculation of the optical density, $D$, a complex number $[11,18]$. The calculation of $D$ requires a measurement of $\rho$ of the bare surface followed by a second measurement after 
the deposition or growth of a surface layer. $\quad D$ is then defined as $\ln \left(\rho_{d} \rho\right)$ where the $o$ subscript refers to the measurement of the original bare surface $[11,18]$.

By standard convention, $\rho$ is expressed in terms of the ellipsometry parameters, $\psi$ and $\Delta$, as $\rho=\tan \psi \mathrm{e}^{\mathrm{i} \Delta}$, where $\psi$ is related to the change in amplitude of the polarized light as a result of the reflection, and $\Delta$ is related to the change in the phase. It follows mathematically then that the real component of $D(\operatorname{Re} D)$ can be expressed as $\ln \left(\frac{\tan \psi_{0}}{\tan \psi}\right)$, whereas the imaginary component $(\operatorname{Im} D)$ is expressed simply as $\Delta_{\mathrm{o}}-\Delta$, where the $o$ subscript once again refers to the measurement of the original bare surface. Garcia-Caurel et al. [11] have shown that the area under the first derivative of an $\operatorname{Im} D$ spectrum (denoted $I m^{\prime} D$ ) is proportional to the concentration of the chemical group corresponding to the absorption at the particular frequency. (Similarly, the second derivative of FTIR transmission spectra results in peak narrowing and easier identification.) As with IR transmission spectra, the intensity of peaks in $\operatorname{Re} D$ and $\operatorname{Im}^{\prime} D$ can be treated quantitatively in determining relative concentrations. The original ellipsometry spectra ( $\psi$ and $\Delta$ ), in contrast, cannot be interpreted in this way.

IR ellipsometry spectra, shown in Figure 1, were successfully obtained from a recentlydeposited mucus trail deposited on a gold-coated substrate. When in the normal ambient atmosphere of the laboratory (relative humidity of ca. 30\%), the trail retains water, as will be discussed later in this paper. The spectra from the bare gold substrate are shown for comparison. 
The raw data shown in Figure 1 was transformed to obtain the $\operatorname{Re} D$ and $\operatorname{Im}$ ' $D$ spectra presented in Figure 2. The transformed data show the presence of certain characteristic absorption bands $[9,19]$ that are expected in IR spectra obtained from pedal mucus. The broad absorption band centered around $3300 \mathrm{~cm}^{-1}$ in the Re $D$ spectrum signifies the presence of hydrogen-bonded water, which is expected since the mucus trail was analyzed shortly after casting and therefore is only partially dry. Note that since this measurement was obtained in the ambient atmosphere, a small amount of $\mathrm{CO}_{2}$ absorption is present in the spectra (as seen in the characteristic doublet around $2300 \mathrm{~cm}^{-1}$ ). [9,19]

Several characteristic absorption bands are present in the $\operatorname{Re} D$ and $\operatorname{Im}^{\prime} D$ spectra, including the $\mathrm{CH}_{2}\left(1443 \mathrm{~cm}^{-1}\right)$ and $\mathrm{CH}_{3}\left(1410 \mathrm{~cm}^{-1}\right)$ deformations of the carbon backbones and C-O stretching (1040 - $\left.1150 \mathrm{~cm}^{-1}\right)$ of the sugar side chains. In general, the $I m$ 'D spectra offer better resolution in comparison to the $R e D$ spectra. For instance, the better peak resolution of the $I m^{\prime} D$ spectrum enables the detection of the amide $\mathrm{N}-\mathrm{H}$ stretching band centered around $3400 \mathrm{~cm}^{-1}$. The small peak in the Re $D$ spectrum around $2900 \mathrm{~cm}^{-1}$ is more fully resolved into the $\mathrm{CH}_{3} / \mathrm{CH}_{2}$ stretches of the carbon backbones in the $\operatorname{Im}^{\prime} D$ spectrum. Furthermore, it is only in the $\operatorname{Im}^{\prime} D$ spectrum that the presence of $\mathrm{C}=\mathrm{O}$ stretching at $1730 \mathrm{~cm}^{-1}$ is resolved. $[9,19]$

For the characterization of the molecular structure of the mucus, the most important absorption bands in the spectra are the protein amide I and II bands at 1651 and 1543 $\mathrm{cm}^{-1}$, respectively. In particular, high resolution de-convolution of the amide I band (the 
Published in Colloids and Surfaces B: Biointerfaces (2004) 33(3-4):251-258

coupling of the $\mathrm{C}=\mathrm{O}$ stretch to the in-plane $\mathrm{N}-\mathrm{H}$ bend and $\mathrm{CN}$ stretch) can reveal a great deal of information about secondary protein structure for single proteins, indicating the presence of $\alpha$-helices, $\beta$-sheets, $\beta$-turns, and non-ordered structures [20]. Despite the complex mixture of protein-carbohydrates in molluscan pedal mucus, analysis of the amide I band by Skingsley et al. [9] has found the presence of $\beta$-sheet and $\beta$-turn structures. However, in the data in Figure 2, the relatively low resolution $\left(16 \mathrm{~cm}^{-1}\right)$ of the original ellipsometry data plus the presence of water in the film (as discussed below) mean that further analysis of the secondary protein structure for the pedal mucus of Achatina marginata is not possible.

Skingsley et al. [9] compared the relative intensity of the amide I and II absorptions for the pedal mucus of Arion ater var. rufus and obtained a ratio of 1:0.68. The pedal mucus of a variety of other species of gastropod in their study showed variation in the relative intensities of the amide I and II bands, which they interpreted as due to variation in the structures of the mucus protein cores [9]. However for the pedal mucus of Achatina marginata shown in Figure 2, the amide I absorption is considerably more intense than the amide II. This may be due to the fact that the film is only partially dry and therefore the amide I absorption will contain a contribution from the strong water deformation absorption around $1645 \mathrm{~cm}^{-1}$.

In the study by Skingsley et al. [9], the contribution of the water was separated from the mucus spectrum by simple subtraction of the water spectrum. However, the interaction of the proteins with water may change the shape of the water absorption band, making the 
Published in Colloids and Surfaces B: Biointerfaces (2004) 33(3-4):251-258

subtraction of a pure water spectrum misleading [21]. It is relevant to note that the relative intensities of the peaks in the infared spectrum of mucins (a primary component of mucus) have been found to vary with water concentration [22]. Additionally, a scaling factor may be needed for the pure water spectrum in order to subtract it from the solution spectrum. Objectively determining this scaling factor is difficult [21]. Thus the differences observed in the relative intensities of amide I and II absorption bands shown in Figure 2 and those reported previously by Skingsley et al. might be, in part, due to the spectroscopic subtraction in the latter. [9]

\section{Ellipsometry Measurement of Water Sorption in Pedal Mucus Trails}

There were obvious changes in the ellipsometry spectra obtained from mucus trail films when the RH in the cell was adjusted. The data could be fitted using an EMA model relying on the optical constants for water and for dry mucus. Ellipsometry analysis found that the thickness of mucus films that had been dried for prolonged times (one or two days) in at atmosphere of $0 \%$ humidity was typically 30 to $35 \mathrm{~nm}$. The solids fraction of the Achatina marginata mucus in its original, native state is not known, but it is usually between 0.95 and 0.99 for most species. Hence, the thickness of the as-deposited mucus trails is estimated to be between 0.5 and $3 \mu \mathrm{m}$. This range of values is much lower than the measurement of 10 to $20 \mu \mathrm{m}$, reported elsewhere [8]. Other researchers have found, however, that the thickness of mucus layers deposited by stationary snails and slugs was significantly greater than what is left by mobile animals [5]. Thus, the fact that the snails were moving while depositing the mucus trail would make it thinner. Furthermore, as we 
show in the next section, the trail thickness is highly sensitive to the ambient relative humidity, and so measurements will vary depending on the conditions.

Simulations using the EMA model (with experimental values of the optical constants for the dry mucus and literature values for water [16]) can be used to demonstrate the capability of IRSE in independently determining film thickness and water content. Figure 3a shows the effect of increasing film thickness with a fixed water content. In regions with no vibrational absorptions, the $\psi$ spectrum is smooth. The total amount of absorption is greater in a thicker film compared to a thinner one, and so there is a downward shift in the $\psi$ spectrum around the characteristic frequencies of water, especially around $3450 \mathrm{~cm}^{-1}$, when the film thickness is increased. $\psi$ decreases by about $0.5^{\circ}$ when the thickness increases from $39 \mathrm{~nm}$ to $59 \mathrm{~nm}$. In the $\Delta$ spectrum, with the same increase in thickness, the gradient increases strongly. The experimental resolution of $\psi$ is approximately $0.02^{\circ}$, and the resolution of $\Delta$ is $0.1^{\circ}$, so the observed differences are significant and can be reliably detected in an experiment.

A change in water content, while keeping the thickness constant, has a very different effect on the ellipsometry spectra, as illustrated in Figure 3b. With a strong absorption, there is a shift in the value of $\psi$, especially around $3450 \mathrm{~cm}^{-1}$, with an increase in water content from 29 vol.\% to 49 vol.\%. The $\Delta$ spectrum, on the other hand, is fairly insensitive to water content. This is an important distinction. The $\Delta$ spectrum is seen to be quite sensitive to film thickness, so when fitting experimental data to a model, obtaining a good fit to $\Delta$ means that the thickness value is reliable. On the other hand, $\psi$ 
is much more sensitive to water content in comparison to $\Delta$. With a correct value of thickness, a good fit to $\psi$ will therefore ensure that a reliable value of water content is obtained.

Simply stated, the simulations reveal that thickness and water content are not strongly correlated. With this in mind, one can have greater confidence in the best fits obtained to the data. An example of a fit is seen in Figure 4. The presence of water in the film is apparent by the increase in the absorptions observed around 1600 and $3450 \mathrm{~cm}^{-1}$. The $\psi$ spectrum shows particular sensitivity to the presence of water. The best-fit EMA model provides a value for the volume fraction of water in the mucus film as being $38 \%$. The film thickness is determined to be $49 \mathrm{~nm}$, compared to a dry thickness of $30 \mathrm{~nm}$. These best-fit values are bracketed by the range of values used for the film thickness and water content in Figures 3a and 3b. IR ellipsometry analysis of the bare gold substrate under varying humidities has shown that the measurement is only very weakly sensitive to the atmospheric water.

Analysis of mucus films at other humidities shows that there is a one-to-one correspondence between thickness change and water concentration as found by ellipsometry. Film thickness typically increased from $30 \mathrm{~nm}$ for dry mucus (in equilibrium with dry air) to $c a$. $65 \mathrm{~nm}$ with a humidity of $84 \%$. Over the same range of humidity, the volume fraction of water is observed to increase from $0 \%$ to $54 \%$. The data thus demonstrate that the volume of water and mucus is conserved and is additive. 
Published in Colloids and Surfaces B: Biointerfaces (2004) 33(3-4):251-258

\section{Water Sorption Isotherm for Pedal Mucus Trails}

There is an extensive literature on the measurement of water vapor sorption in poly(amino acids), peptides and proteins, as has been reviewed elsewhere [23]. There is a qualitative similarity between the water vapor sorption of synthetic glassy polymers (such as poly(vinyl pyrrolidone) and that of biological macromolecules [23,24]. Accordingly, the thermodynamic description of solvent sorption that has been developed for synthetic polymers can, as a first approximation, be applied to natural macromolecules.

At equilibrium, the chemical potential of water in the vapor phase will be equal to the chemical potential of the water that is absorbed in a polymer to form a concentrated polymer solution $[24,25]$. The activity $a$ of the water vapor in the atmosphere is the ratio of the partial pressure of the water vapor divided by the saturated vapor pressure; thus it is apparent that $a=\mathrm{RH} / 100$. At a temperature $T$, the chemical potential of water in the vapor phase is simply expressed as $\mathrm{k} T \ln a$, where $\mathrm{k}$ is Boltzmann's constant.

The chemical potential of a solvent in a polymer solution is given by the well-known Flory-Huggins expression [26]. It has been shown elsewhere [24, 25] that when a polymer is in equilibrium with water in an atmosphere with an activity $a$, the volume fraction of water, $\phi_{\mathrm{w}}$, sorbed in the polymer can be found from the expression

$$
\ln a=\ln \phi_{w}+\left(1-\frac{1}{N}\right)\left(1-\phi_{w}\right)+\chi\left(1-\phi_{w}\right)^{2},
$$

where $\chi$ is the Flory-Huggins polymer-solvent interaction parameter and $N$ represents the number of water molecules that are required to equal the volume of the polymer molecule. ( $N$ is approximately the degree of polymerization and is thus a very large 
number in the case of the large molecules in mucus. The term in brackets can therefore be approximated as unity.) The parameter $\chi$ is a gauge of the interaction energy between the polymer and water at a molecular level. The higher its value, the more hydrophobic is the polymer. A value of $\chi=0.5$ represents a theta-solvent.

It is obvious from Equation 1 that $\chi$ can be found from the dependence of $\phi_{\mathrm{w}}$ on RH. A limitation of this solution model, based on the Flory-Huggins theory, is that it neglects structural changes caused by water sorption, chemical heterogeneity, and the complexity of specific interactions with sorbed water [23]. Furthermore, it is believed that mucus is lightly crosslinked [1], and the effects of crosslinking are not considered in Equation 1. Nevertheless, the model is considered "the best starting point for predicting sorption behavior of biomolecules" [23], and so it is used here in our analysis of the mucus trails. The Flory-Huggins approach has already been applied to determine $\chi$ for poly(vinyl pyrrolidone) (PVP) and water [24, 27] and for poly(DL-lactide) and water [25].

Equation 1 applies to rubbery polymers. A polymer with a glass transition temperature ( $T_{g}$ ) above the temperature of an experiment will pass through its glass transition as solvent is removed. The solvent sorption of a glassy polymer is higher than what is predicted by the standard Flory-Huggins theory [28]. The theory has therefore been modified to consider the compression of the glass matrix as further solvent is removed. Another approach to the problem of solvent sorption in glassy polymers [29] assumes non-additivity of volumes in a two-site adsorption model. 
It is not known for certain if the dry mucus is glassy at the temperature of the experiment. The $T_{g}$ of mucin, which is a major constituent of mucus, has been determined, however, and offers some insight. Davies and Viney [30] reported that the $T_{g}$ of pig gastric mucus is $25^{\circ} \mathrm{C}$, which is the temperature of our measurements. Therefore, we are led to expect that the snail pedal mucus is rubbery during our measurements and that the FloryHuggins theory is not invalidated by the onset of vitrification at low water concentrations.

Measurements of water sorption in the same mucus film as a function of $\mathrm{RH}$ were used to characterise hydrophilicity, using $\chi$ as a gauge for comparison to other substances. The results of one such experiment are plotted in Figure 5. Water uptake increases strongly when $\mathrm{RH}$ increases above $60 \%$. When $\mathrm{RH}=84.3 \%$, the volume fraction of water increases to 53\%. This observed trend was reproduced in other experiments. The data are described adequately using Equation 1 and varying $\chi$ to obtain the best fit. The simulated adsorption isotherm agrees with the experimental data over the entire humidity range. As expected from the comments in the previous paragraph, there is no evidence for a "shoulder" in the isotherm at lower humidities, as is found when a rubbery polymer vitrifies. A $\chi$ value of $0.54 \pm 0.1$ is obtained from the best fit, indicating that the mucus is very hydrophilic.

To put this result in context, it is helpful to compare to $\chi$ values obtained elsewhere for synthetic polymers. Maccarini [27] conducted gravimetric measurements on thin films of PVP using a quartz-crystal microbalance and found $\chi=0.51 \pm 0.03$ for PVP/water. This measurement of $\chi$ for PVP thin films agrees well with the value obtained for the same 
material in the bulk form [24]. In contrast, poly(DL-lactide) (in thin films) was found to be much less hydrophilic, with a value of $\chi=3.50$ for its interaction with water [25]. Our measurements indicate that the molecules in the mucus, which are initially deposited from water, retain their hydrophilicity after their deposition. Furthermore, crosslinking in the mucus trail is not particularly high, or otherwise the water uptake would be restricted, as predicted by the Flory-Rehner theory [26], and the apparent $\chi$ value would be larger than 0.5 because there would have been less swelling in vapor.

It is also relevant to point out that the measurement reported here is from a process in which the humidity began at $0 \%$ and was increased during the measurement. Future work will study moisture uptake during cycles of the humidity from low to high and then back again. Elsewhere [31], experiments on mammalian mucins have found a strong hysteresis in water vapor sorption. This result was attributed to the denaturing of the protein moities of the mucin creating a structural change with a rearrangement of the hydrophilic carbohydrate moieties.

Since mucin is a primary constituent of mucus, it is relevant to consider some of the characteristics of mucin here. In other work, mucin coatings have been shown to be effective in suppressing bacterial adhesion to polymer surfaces [32, 33]. They therefore offer a means by which to reduce the risk of microbial infections on polymers used in temporary or permanent implants. The hydrophilicity of the mucus trails might similarly impart anti-bacterial properties to protect the snail. 
Published in Colloids and Surfaces B: Biointerfaces (2004) 33(3-4):251-258

Mucin consists of large, linear molecules with a hydrophobic backbone on which there are hydrophilic oligosaccharide clusters [32]. The molecule therefore has an amphiphilic character that enables it to adsorb onto hydrophobic surfaces to create a hydrophilic coating. It is therefore insightful to compare the absorption isotherms from the mucus trails to those obtained from proteins in previous work reported elsewhere. For instance, a water sorption isotherm was obtained from lysozyme gravimetrically [34]. At lower RH values, the results were consistent with the predictions of a group-contribution model. At a temperature of $35{ }^{\circ} \mathrm{C}$ and $\mathrm{RH}=80 \%$, water uptake was approximately $26 \mathrm{wt} . \%$ [32]. Isotherms obtained using Raman spectroscopy on thick (1.5 to $2.5 \mu \mathrm{m})$ films of bovine serum albumin show an uptake of about 22 wt.\% at the same RH [35]. The water sorption in these two proteins at $\mathrm{RH}=80 \%$ is about one-half as much as observed in the mucus films. These results suggest that the hydrophilicity of the carbohydrates and polysaccharides dominates over any hydrophobicity of the protein components.

The adsorption of mucin onto polymer surfaces requires a technique to measure the amount deposited. SDS-PAGE electrophoresis has been used for this purpose [32]. IRSE offers a simpler and faster means of characterization for mucin coatings on a variety of substrates, including polymers. We have already confirmed that a mucus trail of the giant African land snail can be detected and identified when deposited onto the surface of a poly(dimethyl siloxane) coating. Raman spectroscopy has been applied previously to measure water vapor sorption in thick protein films [35], but this technique places restrictions on the refractive index of the substrate and requires a prism to be 
Published in Colloids and Surfaces B: Biointerfaces (2004) 33(3-4):251-258

placed in contact with the film to couple light into it. IRSE offers much greater flexibility in the choice of substrate surface.

\section{Conclusions}

Infrared ellipsometry has been used to identify the presence of mucus trails deposited by the giant African land snail (Achatina marginata) onto a solid substrate. The ellipsometry technique is quick and can reliably identify the major chemical constituents in the thin layers (typically $30 \mathrm{~nm}$ thick when dry). It is likely that the technique could discriminate between the mucus of other species of land or marine snail, as has been likewise found for IR spectroscopy. The ellipsometry spectra reveal the presence of characteristic chemical groups for gastropod mucus, including $\mathrm{CH}_{2}, \mathrm{CH}_{3}, \mathrm{CO}, \mathrm{C}=\mathrm{O}$, and most importantly - the amide I and II bands.

The thickness and water content of the mucus trail of the Achatina marginata snail are a strong function of the relative humidity. The equilibrium water vapor sorption isotherm of the mucus trail can be described using a solution model with a Flory-Huggins polymer/solvent interaction parameter of $\chi=0.54 \pm 0.1$. This value is similar to what is obtained for very hydrophilic synthetic polymers, such as poly(vinyl pyrrolidone).

The fit to the Flory-Huggins theory enables the prediction of water uptake in mucus for a given value of the ambient relative humidity. For instance, at a typical value of $30 \%$ $\mathrm{RH}$, the mucus contains approximately 10 vol.\% water when in equilibrium with the atmosphere. Thus, the mucus trail remains hydrated under typical ambient conditions. 
Published in Colloids and Surfaces B: Biointerfaces (2004) 33(3-4):251-258

The observed water sorption is much greater than what has been found in simple proteins (i.e. lysozyme and bovine serum albumin). The hydrophilicity of the mucus might influence the trailing and homing mechanisms of snails, and it might also provide antibacterial protection for the snail. Future measurements can be performed on other species of snail, including marine species, and on other substrates, including polymers.

\section{Acknowledgments}

We appreciate the technical support of J.-W. Brown (University of Surrey). Funding for the ellipsometer and for T.R.E.S. was provided by the UK Engineering and Physical Sciences Research Council. 
Published in Colloids and Surfaces B: Biointerfaces (2004) 33(3-4):251-258

\section{References}

1. M. Denny in The Mollusca, Vol. 1: Metabolic Biochemistry and Molecular Biomechanics, P.W. Hochachka, ed., Academic Press: London (1983), Ch. 10, pp. 431437.

2. M. Denny, Nature, 285 (1980) 160.

3. T.J. Trott and R.V. Dimock, Mar. Behav. Physiol., 5 (1978) 91.

4. J.M. Cottrell, I.F. Henderson, J.A Pickett, D.J. Wright, Comp. Biochem. Physiol. B Biochem. Molec. Biol., 104 (1993) 455.

5. S.P. Holmes, A. Cherrill, and M.S. Davies, J. Marine Biol. Assoc. U.K., 82 (2002) 131.

6. D.J. Gerhart, D. Rittschof, I.R. Hooper, K. Eisenman, A.E. Meyer, R.E. Baier, and C. Young, Biofouling, 5 (1992) 251.

7. M. Malmsten, E. Blomberg, P. Claesson, I. Carlstedt, and I. Ljusegren, J. Coll. Interf. Sci., 151 (1992) 579.

8. M. Denny, Science, 208 (1980) 1288.

9. D.R. Skingsley, A.J. White, A. Weston, J. Moll. Stud., 66 (2000) 363.

10. T. Watanabe, Fukushima J. Med. Sci., 22 (1976) 245.

11. E. Garcia-Caurel, B. Drévillon, A. De Martino, and L. Schwartz, Appl. Opt., 41 (2002) 7339.

12. D. Tsankov, K. Hinrichs, A. Röseler, and E.H. Korte, Phys. Stat. Sol. A, 188 (2001) 1319.

13. W.-L. Chen, K.R. Shull, T. Papatheodorou, D.A. Styrkas, J.L. Keddie, Macromolecules, 32 (1999) 136. 
Published in Colloids and Surfaces B: Biointerfaces (2004) 33(3-4):251-258

14. A. Wexler and S. Hasegawa, J. Res. Nat. Bur. Stand., 53 (1954) 19.

15. D.E. Aspnes, Phys Rev B, 33 (1986) 677.

16. E.D. Palik, Handbook of Optical Constants, Academic Press: London (1991), pp. 1073-1076.

17. G.E. Jellison, Appl. Opt., 30 (1991) 3354.

18. S. Vallon, B. Drévillon, and F. Poncin-Epaillard, Appl Surf. Sci., 108 (1997) 177.

19. D.H. Williams and I. Fleming, Spectroscopic Methods in Organic Chemistry, McGraw-Hill: London (1989), pp. 29-62.

20. W.K. Surewicz and Mantsch, Biochimica et Biophysica Acta, 952 (1988) 115.

21. W.K. Surewicz, H.H. Mantsch and D. Chapman, Biochemistry, 32 (1993) 389.

22. F. Liu, J.-G. Wu, R.D. Soloway, H. Guo, D.K. Martini, N. Xu, and G.-X. Xu, Mikrochim. Acta (Wien), 1 (1988) 357.

23. S.L. Shamblin, B.C. Hancock, G. Zografi, Eur. J. Pharmaceutics Biopharm., 45 (1998) 239.

24. B.C. Hancock and G. Zografi, Pharmaceutical Research, 10 (1993) 1262.

25. J.S. Sharp, J.A. Forrest, and R.A.L. Jones, Macromolecules, 34 (2001) 8752.

26. P.J. Flory, Principles of Polymer Chemistry, Cornell University Press: Ithaca, New York (1953). 27. M. Maccarini, Ph.D. thesis, University of Sheffield (2002) Chapter 6.

28. L. Leibler and K. Sekimoto, Macromolecules, 26 (1993) 6937.

29. J.S. Ventras and C.M. Ventras, Macromolecules, 24 (1991) 2404.

30J.M. Davies and C. Viney, Thermochimica Acta, 315 (1998) 39.

31. F. Bettelheim and A. Block, Biochimica et Biophysica Acta, 165 (1968) 405.

32. L. Shi, R. Ardehali, K.D. Caldwell, and P. Valint, Coll. Surf. B: Biointerfaces, 17 (2000) 229. 
Published in Colloids and Surfaces B: Biointerfaces (2004) 33(3-4):251-258

33. L. Shi, R. Ardehali, P. Valint, and K.D. Caldwell, Biotechnol. Lett., 23 (2001) 437.

34. J.D. Leeder and I.C. Watt, J. Coll. Interf. Sci., 48 (1974) 339.

35. B.A. Bolton and J.R. Scherer, J. Phys. Chem., 93 (1989) 7635. 
Published in Colloids and Surfaces B: Biointerfaces (2004) 33(3-4):251-258

\section{Figure Captions}

Figure 1. IR ellipsometry spectra (both $\psi$ (top) and $\Delta$ (bottom)) obtained from a bare gold-coated substrate (light line) and from a partially-dried mucus film in the ambient atmosphere on the same substrate (heavy line).

Figure 2. $\operatorname{Re} D$ (solid lines) and $I m$ 'D spectra (dashed lines) obtained from a partiallydried mucus trail over (a) the upper range of wavenumbers and (b) the lower range of wavenumbers.

Figure 3. Simulations to illustrate the sensitivity of $\psi$ and $\Delta$ to changes in film thickness and in water content. (a) Simulations of spectra for a film containing 39 vol.\% water and having two different thicknesses: $39 \mathrm{~nm}$ (light line) and $59 \mathrm{~nm}$ (heavy line); (b) Simulations of spectra for a $49 \mathrm{~nm}$ thick film containing two different water contents: 29 vol.\% (light line) and 49 vol.\% (heavy line).

Figure 4. Ellipsometry spectra ( $\psi$ (top) and $\Delta$ (bottom)) obtained from a mucus film in an atmosphere with $\mathrm{RH}=74.3 \%$. The light line is the best fit to the data (shown with the heavy line) using an EMA model for a 49 nm thick film containing 39 vol.\% water.

Figure 5. Equilibrium water vapor sorption isotherm for a mucus film on a solid substrate, as determined from IR ellipsometry measurements. The line is a prediction 
Published in Colloids and Surfaces B: Biointerfaces (2004) 33(3-4):251-258

based on the Flory-Huggins expression using a best-fit value for the polymer/solvent interaction parameter of $\chi=0.54$. 
Published in Colloids and Surfaces B: Biointerfaces (2004) 33(3-4):251-258
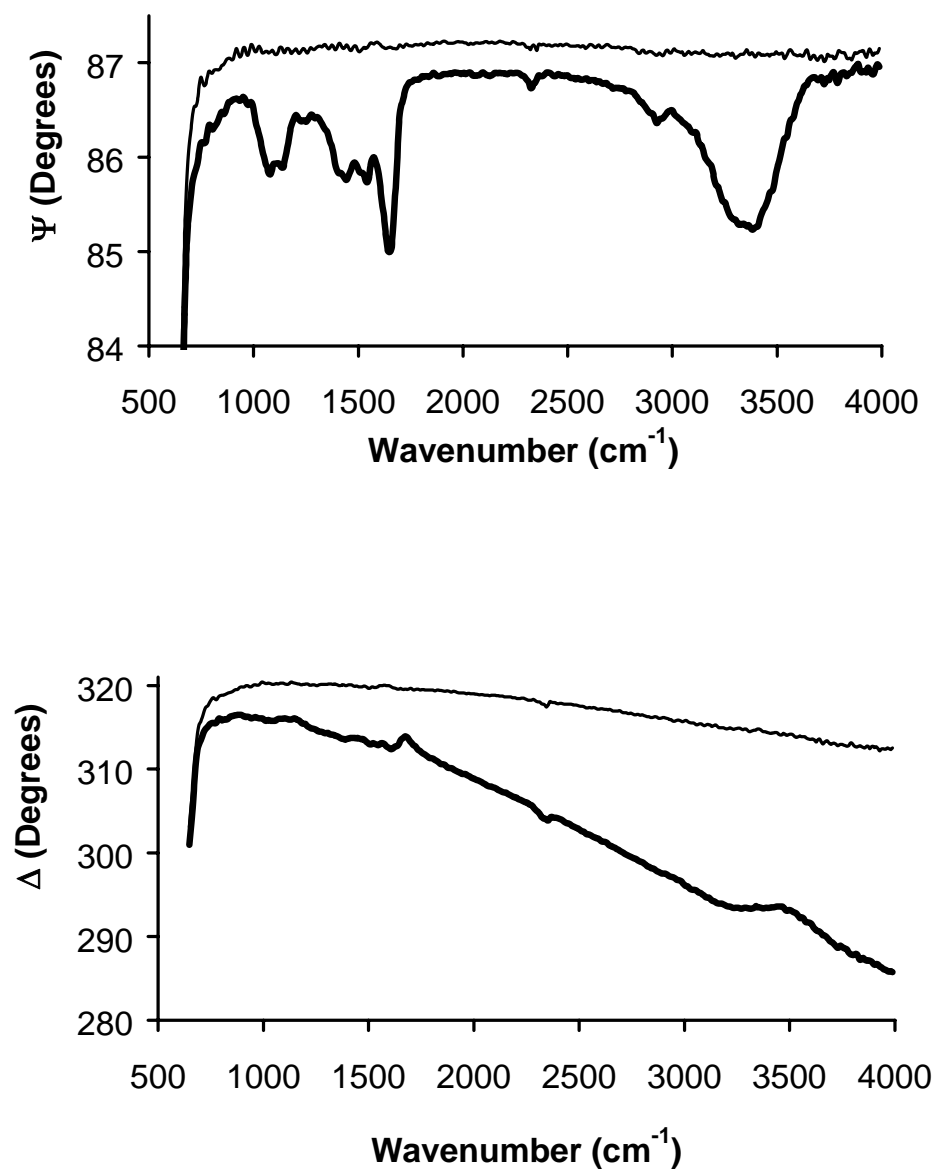

Figure 1 
Published in Colloids and Surfaces B: Biointerfaces (2004) 33(3-4):251-258

(a)

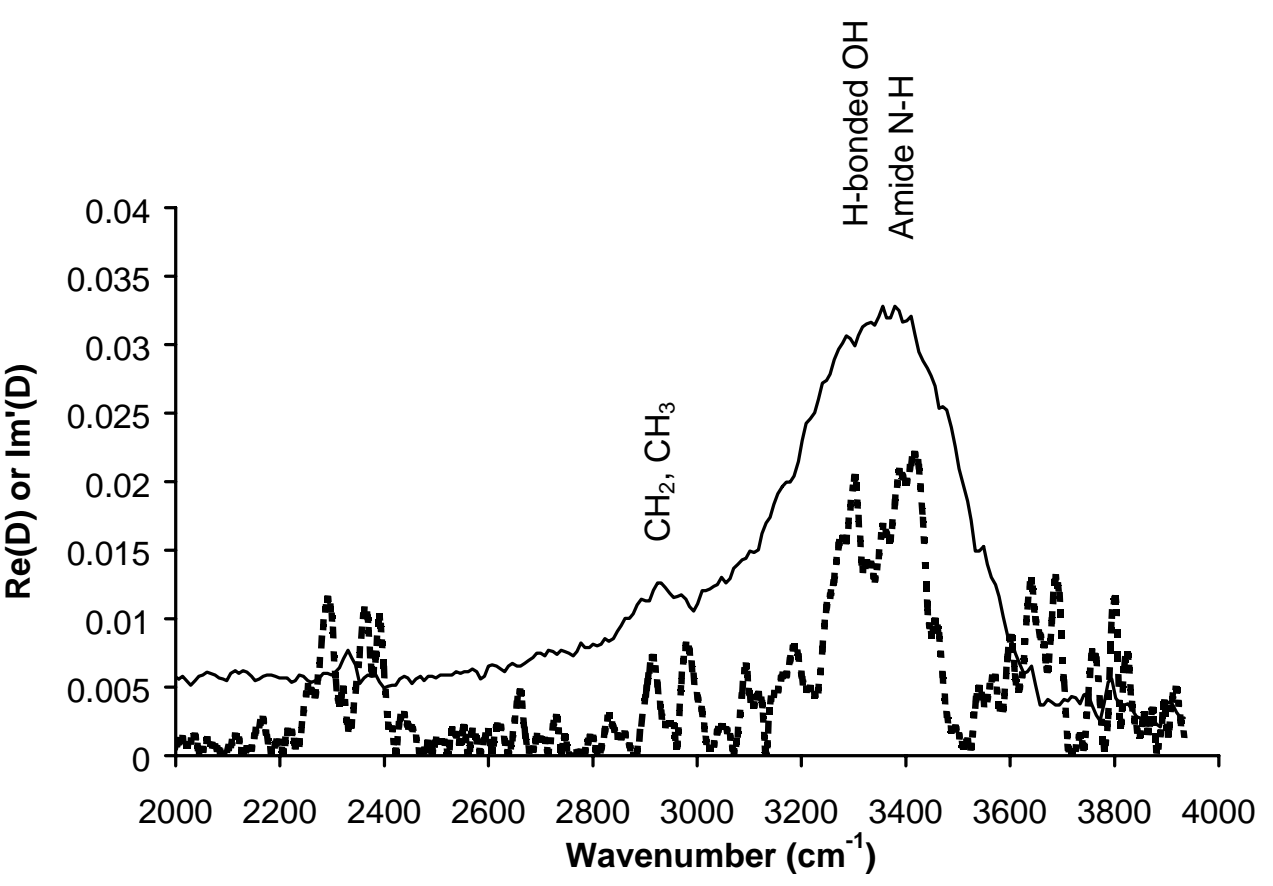

(b) 0.05

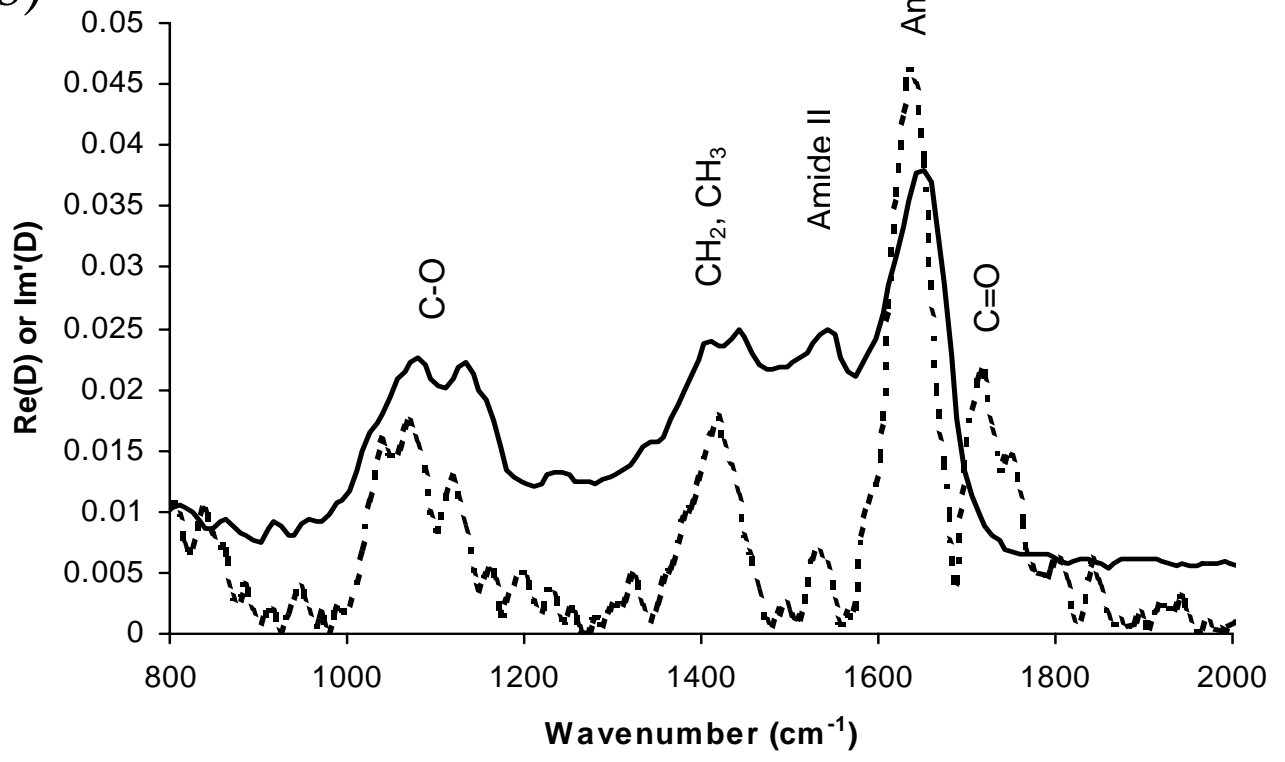

Figure 2 
Published in Colloids and Surfaces B: Biointerfaces (2004) 33(3-4):251-258

(a)
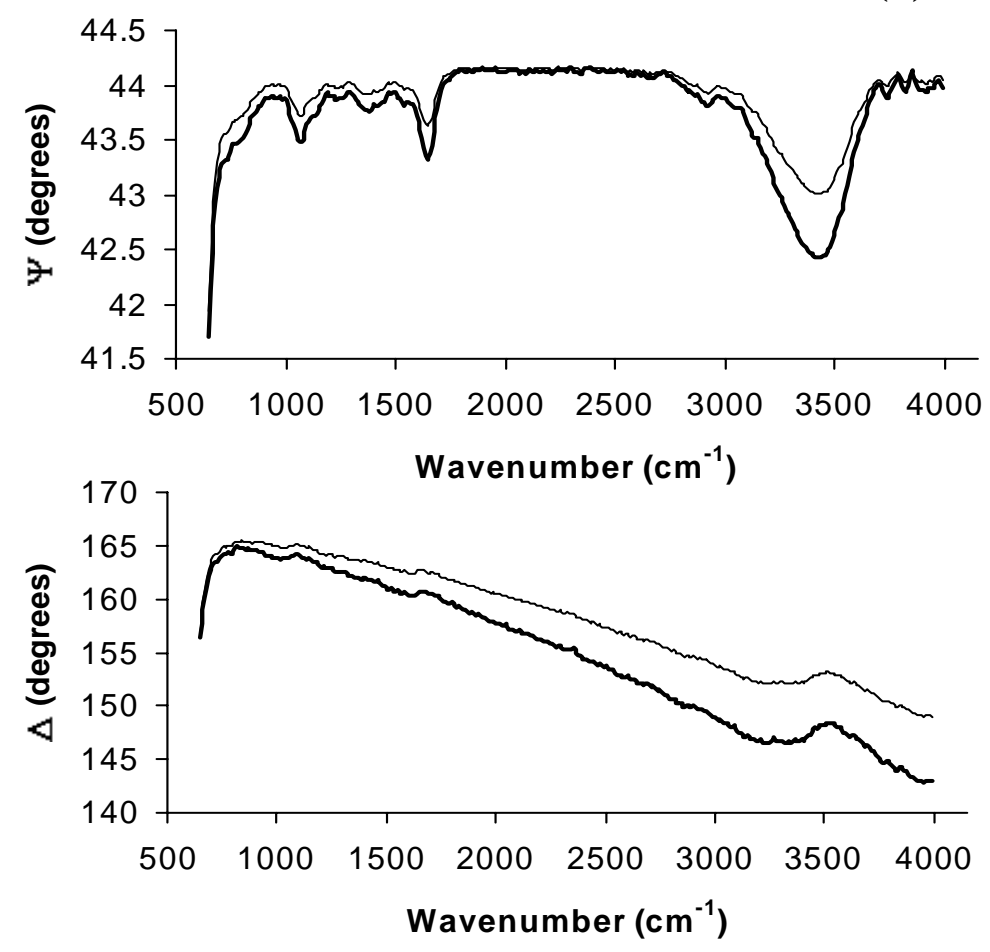

(b)
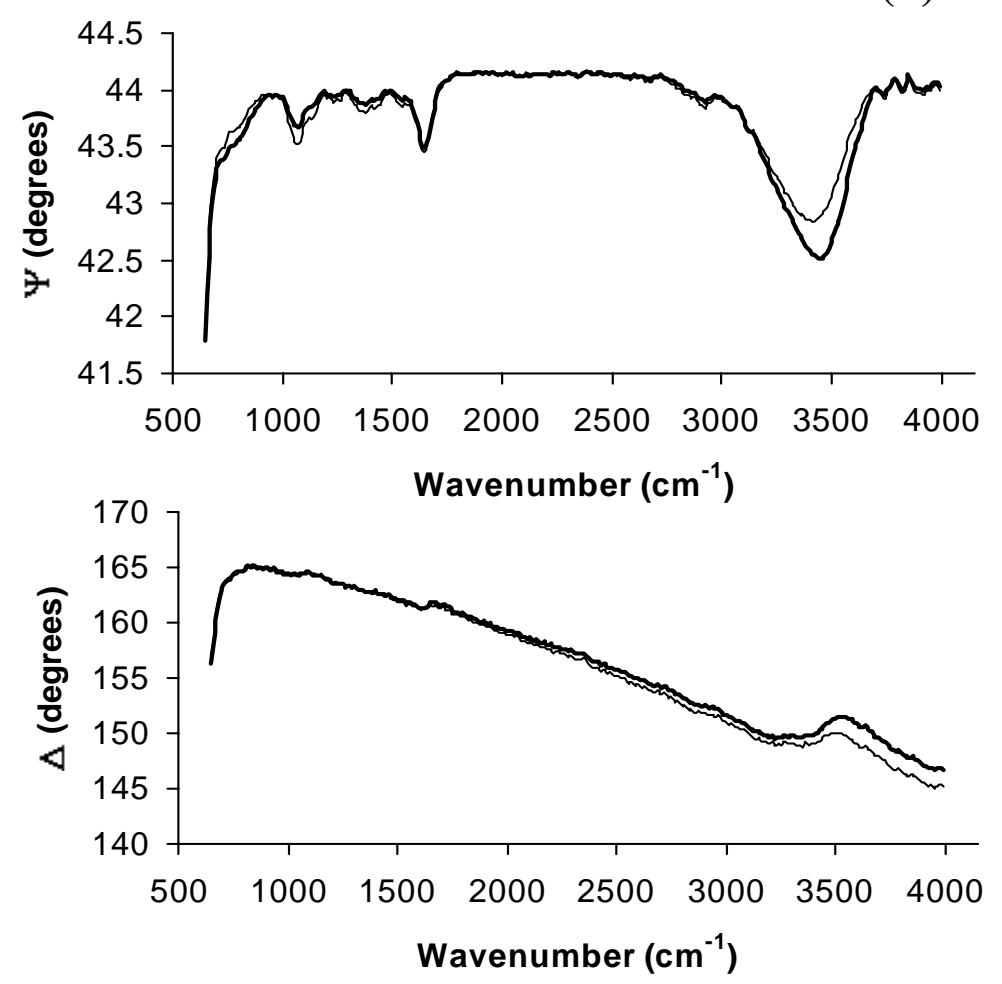

Figure 3 
Published in Colloids and Surfaces B: Biointerfaces (2004) 33(3-4):251-258
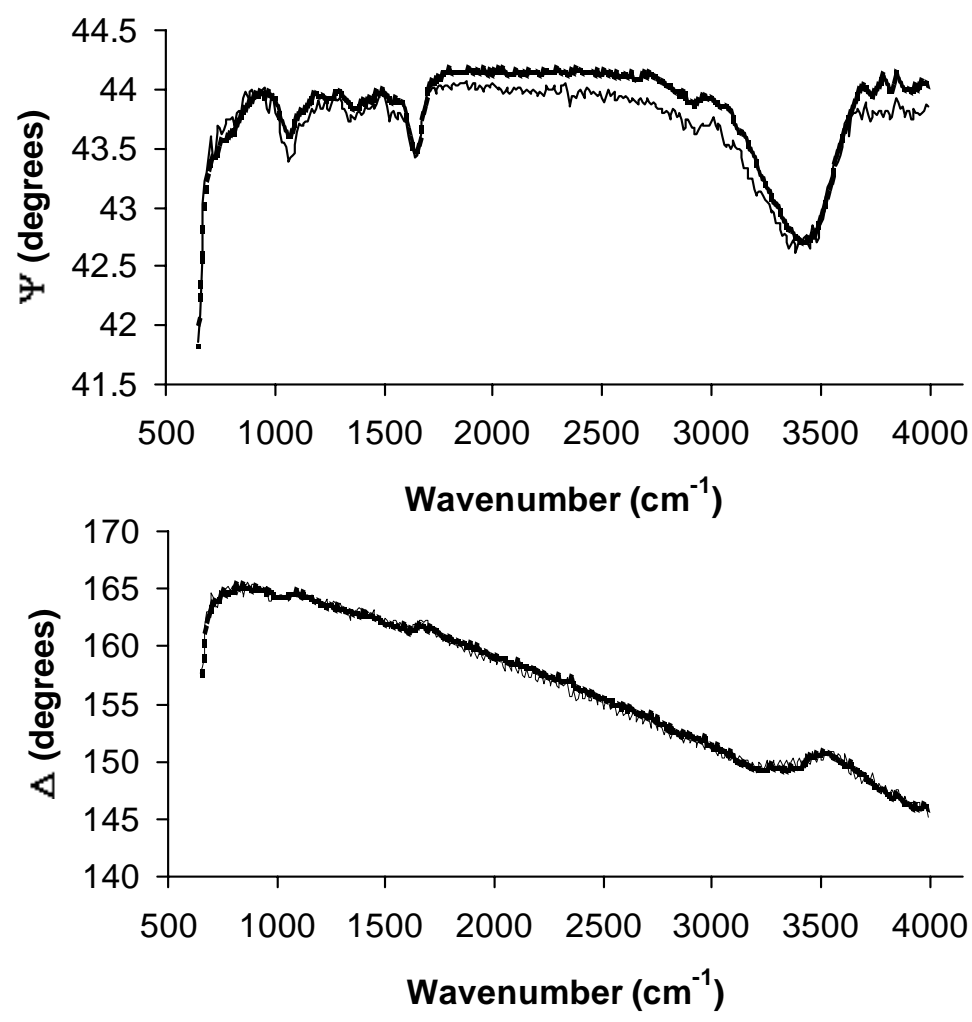

Figure 4 
Published in Colloids and Surfaces B: Biointerfaces (2004) 33(3-4):251-258

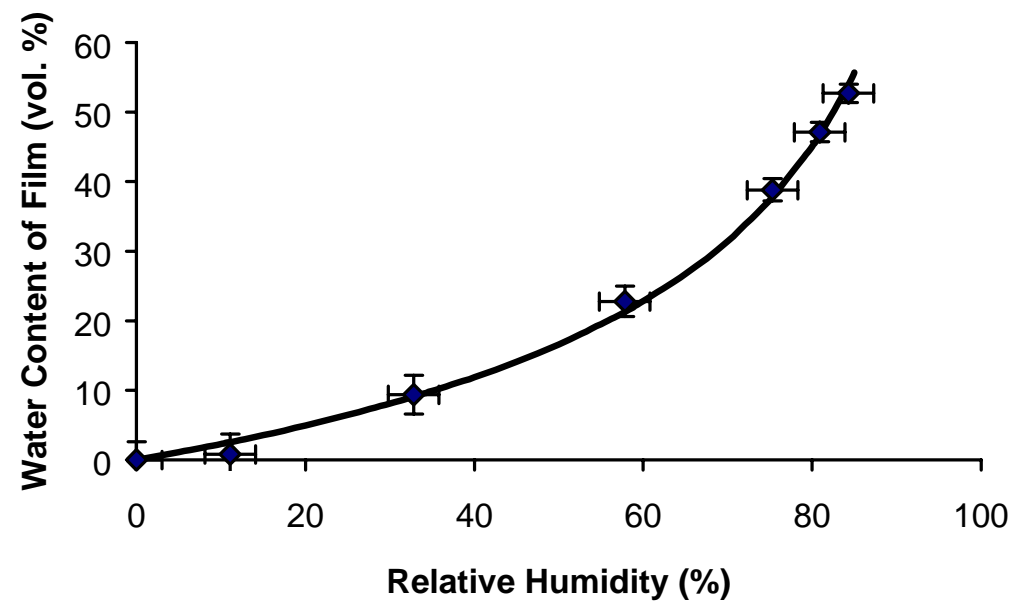

Figure 5 